\title{
Oblique cross-waves in horizontally vibrated containers
}

\author{
José M Pérez-Gracia ${ }^{1}$, Jeff Porter ${ }^{1}$, Fernando Varas ${ }^{1,2}$ and \\ José M Vega ${ }^{1}$ \\ ${ }^{1}$ ETSI Aeronáuticos, Univ. Politécnica de Madrid, Pl. Cardenal Císneros, 3, 28040 \\ Madrid, Spain \\ ${ }^{2}$ EI Telecomunicación, Univ, de Vigo, Campus Marcosende, E-36310 Vigo, Spain \\ E-mail: fernando.varas@upm.es
}

Received 14 November 2013, revised 14 November 2013

Accepted for publication 17 February 2014

Published 22 April 2014

Communicated by E Knobloch and A Oron

\begin{abstract}
The excitation of subharmonic waves on the free surface of a horizontally vibrated, rectangular container of liquid is considered and the properties of threshold patterns are obtained and discussed. These waves are generally quasiperiodic and oblique (not aligned with the container walls). The parametric forcing mechanism generated by the harmonic oscillatory bulk flow is assumed to dominate over that associated with harmonic surface waves and a linear theory recently developed by the authors [Perez-Gracia et al $2014 \mathrm{~J}$. Flid Mech. 739 196-228] is used to compute both the threshold forcing amplitude and the pattern orientation. Two distinct regimes are considered: (1) large containers where the subharmonic waves generated at each endwall do not interact appreciably and (2) smaller containers where interaction occurs. The nature of the critical eigenfunction is examined in each case. and a contrast drawn between pure 2:1 resonance and the general case of quasiperiodic instability.
\end{abstract}

(Some figures may appear in colour only in the online journal)

\section{Introduction}

Vibrated liquids have long attracted experimental and theoretical attention. especially so since the seminal work of Faraday (1831). Such interest reflects both their practical importance over a wide range of scientific and engineering applications such as liquid storage. mixing. and structure formation, and the rich variety of phenomena that can arise. Fluid properties, system size, timescales, symmetry properties, can vary enormously from one application to the next. and there are numerous possible effects to consider. Vertical vibration. for instance, can delay 
convection (Gresho and Sani 1970), suppress the Rayleigh-Taylor instability (Wolf 1969), induce mean flows (Vega et al 2001), and affect heat or mass transfer. In addition to these effects (see, e.g., Nicolas et al 1998), horizontal vibration can drive sloshing and other types of resonant and near-resonant mode dynamics at low frequencies (Miles 1984, Funakoshi and Inoue 1988, Feng 1997) and produce quasistatic structures called frozen waves (Talib et al 2007) at intermediate frequencies on the interface between two immiscible fluids.

Theoretical efforts to explain this range of phenomena are necessarily broad in method and scope. Nonetheless, there are certain simplifying assumptions that appear repeatedly in theoretical analyses, such as treating the forcing function as monochromatic with constant amplitude and direction. Except in precisely controlled experiments, environmental vibrations are generally broad-band and vary in both magnitude and direction. However, it may often be that the support structure surrounding the fluid, with its own mechanical resonances, transmits acceleration that is narrow-band and centered on specific frequencies and the monochromatic assumption can thus be regarded as a reasonable idealization. If multi-frequency vertical forcing is considered, on the other hand, complex states such as quasipatterns and superlattice patterns can arise (see, e.g., Arbell and Fineberg 2002, Topaz et al 2004 and references therein) due to the influence of additional modes. The assumption of a constant axis of vibration is similarly widespread and simplifies analysis. Recent work, however, points to the new phenomena that can arise with circular (Ivanova et al 2001) or two-axis acceleration (Bestehorn et al 2013).

Another common assumption in vibrated fluid systems is of spatially uniform forcing. This is justified in the usual vertically forced Faraday problem, where an open container of liquid is vibrated in purely vertical fashion and capillary effects at the boundaries are ignored. The theoretical advantages of a uniform parametric forcing mechanism help explain why vertically forced Faraday waves have received more attention than horizontally forced systems. The assumption of uniform forcing is invalid, however, in more generic situations. Capillary effects in the Faraday problem, for example, generate harmonic waves near the boundaries that break the uniformity of the flat state. Horizontal vibration leads to vertical motion at the boundaries and a spatially nonuniform bulk flow (Varas and Vega 2007), complicating analysis.

Confined horizontally vibrated fluids can be viewed as a counterpoint to the well-studied nongeneric case of uniform forcing. These systems possess harmonic waves, driven by the motion of the boundaries, that appear with arbitrarily small forcing. Subharmonic waves appear at higher forcing (see Miles and Henderson 1990 and references therein) and, although these are otherwise analogous to vertically forced Faraday waves, they are concentrated near the moving boundaries (wavemakers) that produce them. These waves are traditionally called cross-waves due to their preferred orientation in gravity wave experiments. In deep containers they are driven by an oscillating bulk flow, as identified in a two-dimensional case by Varas and Vega (2007), which is concentrated in a region near the vibrating walls. The dependence of vibrated fluid phenomena on nonuniform extended forcing mechanisms like this is something that has only recently been investigated in detail (Varas and Vega 2007, Porter et al 2012, Moisy et al 2012, Perez-Gracia et al 2014), despite the fact that such mechanisms are more generic than uniform forcing.

The subharmonic cross-wave instability is a robust transition that is easily observed in both wavemaker experiments (Schuler 1933, Lin and Howard 1960, Barnard and Pritchard 1972, Underhill et al 1991) and horizontally vibrated systems (Porter et al 2012). Like vertically forced Faraday waves, cross-waves arise from a parametric instability (Garrett 1970) and can exhibit complex behaviour such as modulations, mode interactions, and chaotic dynamics. The most influential theory of cross-waves was developed by Jones (1984), who derived a nonlinear Schrödinger (NLS) equation to describe their slow spatial and 

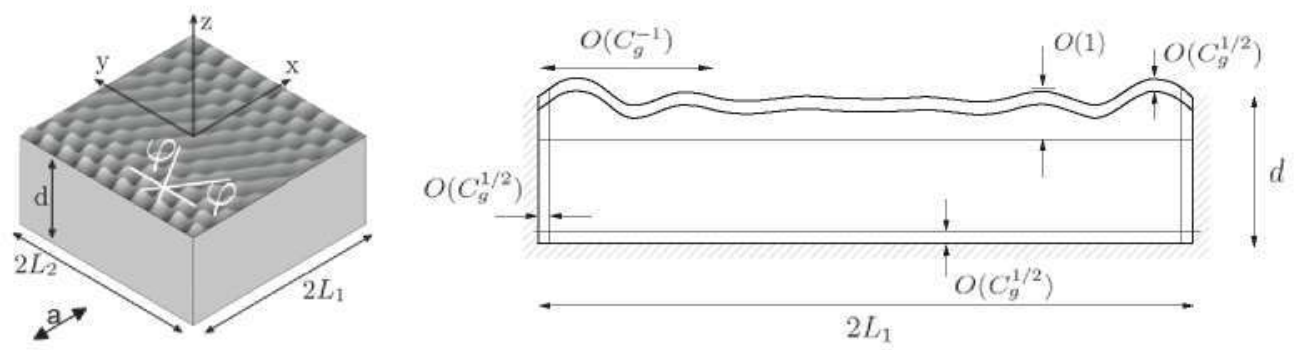

Figure 1. Left: sketch of the vibrated container. Right: the distinguished regions of the flow structure.

temporal evolution. Subsequent work included viscous effects (Lichter and Chen 1987, Bernoff et al 1989, Shemer and Kit 1989) and mode interactions (Ayanle et al 1990). Although these models were compared with some success to cross-wave experiments (Lichter and Bernoff 1988, Kit and Shemer 1989, Shemer and Kit 1989, Underhill et al 1991), it is important to note that these motivating experiments were all in the gravity wave regime and that the NLS models rely on assumptions appropriate to that limit. One such assumption is that cross-waves vary slowly in the downstream direction compared both to the crosswise wavelength and to the extent of the localized forcing. This permits a separation of lengthscales that places the forcing term in the homogeneous boundary condition of the NLS equation. Generally, such a separation of scales occurs when the wavemaker penetrates, at most, a distance comparable to the wavelength. If the wavemaker is deep, the associated forcing mechanism will extend further into the interior and its spatial dependence should be taken into account.

We consider the general problem of a horizontally vibrated container, including the gravity-capillary regime explored by more recent experiments (Taneda 1994, Porter et al 2012, Moisy et al 2012) where the spatial dependence of the parametric forcing mechanism producing cross-waves cannot be ignored. The theoretical analysis of this problem has recently been carried out by the authors (Perez-Gracia et al 2014) and can be seen as a generalization of standard cross-wave theory that permits more general classes of subharmonic solutions. In particular, Perez-Gracia et al (2014) showed that these waves are generally quasiperiodic (that is, not strictly $2: 1$ subharmonic) and oblique (not aligned with the container walls). For the sake of completeness, this analysis is outlined briefly at the end of section 2 following a statement of the problem and its principal assumptions, and a discussion of the competing forcing mechanisms for subharmonic waves. In this paper, we use this theory to systematically investigate the role of the different parameters and provide detailed calculations that could be of interest for experiments with horizontally vibrated systems. More precisely, we focus on the qualitative difference between two experimentally relevant limits related to the strength of the interaction between subharmonic waves generated at opposing endwalls. In the first limit, considered in section 3, the container is assumed to be large enough (compared to the viscous decay length) that the interaction between the subharmonic waves generated at each endwall is negligible. In the second limit, considered in section 4 , the container is smaller and interaction between both subharmonic wavetrains must be considered. 


\section{Subharmonic instabilities in horizontally vibrated containers}

\subsection{Problem statement and assumptions}

We consider a brim-filled container of liquid with rectangular cross section that is subject to horizontal vibration. (see the left part of figure 1). The dimensional forcing amplitude (aligned as in figure 1 with one pair of the container walls) is denoted by $a^{*}$. the forcing frequency by $2 \omega^{*}$. the depth by $d^{*}$, the length along the direction of vibration by $2 L_{1}^{*}$, and the length in the transverse direction by $2 L_{2}^{*}$.

Subharmonic waves will exhibit a wavenumber close to $k^{*}$ given by the inviscid dispersion relation

$$
\left(\omega^{*}\right)^{2}=g k^{*}+\frac{\sigma}{\rho}\left(k^{*}\right)^{3}
$$

where $g$ is the gravitational acceleration, $\sigma$ the surface tension, and $\rho$ the fluid density. These three parameters will be kept constant in the analysis below.

The principal assumptions made in this paper are the following.

(i) The container is deep and wide compared to the wavelength

$$
d=k^{*} d^{*} \gg 1 \quad L_{1}=k^{*} L_{1}^{*} \gg 1 \quad L_{2}=k^{*} L_{2}^{*} \gg 1
$$

(ii) The damping of gravity-capillary waves due to viscosity and measured by the Ohnesorge number $C_{g}$ (representing the ratio of viscous to inertia terms in the momentum conservation equation) is small:

$$
C_{g}=\frac{\mu\left(k^{*}\right)^{2}}{\rho \omega^{*}} \ll 1,
$$

where $\mu$ denotes the viscosity. Although gravity-capillary waves can thus be considered nearly inviscid (i.e., viscous dissipation at the scale of the wavelength can be neglected), they will, in general. exhibit spatial modulation because of viscous damping (experienced as the wave travels inward along the length of the container, away from the driving endwalls). Since the amplitude of the gravity-capillary waves decays exponentially with a factor proportional to $C_{g}$, the size of the interaction between subharmonic waves excited near opposite endwalls will depend on the product of $C_{g}$ and the container length $L_{1}$.

(iii) The contact line is fixed at the brim of the container. This assumption (which can be imposed experimentally using either sharp edges or hydrophobic/oleophobic coatings) is important to avoid the uncertainty associated with a dynamic contact line, whose modeling is still not well understood (Snoeijer and Andreotti 2013).

Note that no assumption is made about the gravity-capillary balance. This balance can be measured by the nondimensional number $S$ defined by

$$
S=\frac{\sigma\left(k^{*}\right)^{2}}{\sigma\left(k^{*}\right)^{2}+\rho g},
$$

which can take any value from $S=0$ (the pure gravity wave limit) to $S=1$ (the pure capillary wave limit). 

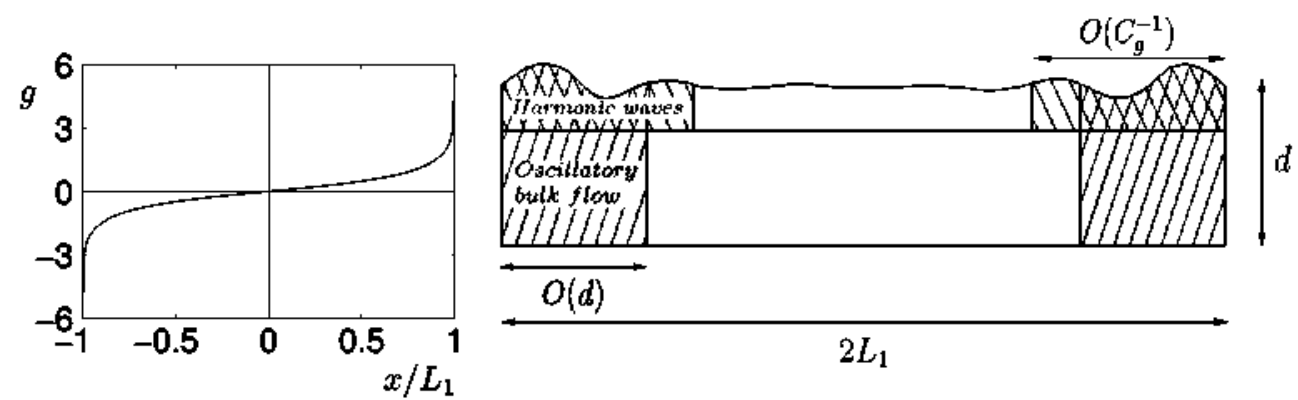

Figure 2. Left: vertical pressure gradient, $g(x)$, associated with the OBF for $d=L_{1}$. Right: forcing regions associated with harmonic surface waves and the $\mathrm{OBF}$.

The structure of the flow under the above assumptions, which lead to a separation of scales, can be divided into distinct regions. illustrated in the right part of figure 1:

(i) Viscous boundary layers attached to the bottom of the container, the walls of the container and the free surface. These boundary layers have a thickness (nondimensionalized by the wavenumber $k^{*}$ ) of the order of $\sqrt{C_{g}}$. Vorticity is also, in general, confined within these layers.

(ii) A layer beneath the free surface that is affected by the (propagation and reflection of) surface waves. This layer is thicker than the viscous one, of the order of the characteristic wavelength (i.e.. $O(1)$ in nondimensional units).

(iii) An interior region that excludes the above boundary layers, referred to here as the bulk region.

\subsection{Harmonic forcing terms}

In comparison to the case of a vertically vibrated container. horizontal vibration produces a more complex harmonic (i.e., synchronous) flow. The separation of scales associated with the limit considered in this paper leads to two different harmonic components. These are

- A pair of counter-propagating wavetrains, traveling inward from the opposing endwalls. These are surface waves generated by the movement of the (upper part of the) endwalls. They penetrate (from the endwalls) a distance determined by the damping. since the exponential decay factor is proportional to $C_{8}$. Their crests are parallel to the endwalls and their characteristic wavelength is given by the dispersion relation (1) with the forcing frequency $2 \omega^{\star}$ replacing the subharmonic frequency $\omega^{\star}$. These harmonic wavetrains were described (including a weakly nonlinear analysis) in Varas and Vega (2007).

- An oscillatory bulk flow (OBF). This flow is generated in the bulk region by the oscillation of the (entire surface of the) endwalls. The leading order term of the hydrostatic stagnation pressure associated with the $\mathrm{OBF}, P_{O B F}$, is given by (details can be found in Varas and Vega 2007 and Perez-Gracia et al 2014)

$$
P_{O B F}(x, y, z, t)=a \cos (2 t) \Phi(x, z)
$$




$$
\Phi(x, z)=\frac{32 d}{\pi^{2}} \sum_{m>0,0 d d} \frac{\sinh (m \pi x /(2 d)) \cos (m \pi(z+d) /(2 d))}{m^{2} \cosh \left(m \pi L_{1} /(2 d)\right) \sin (m \pi / 2)},
$$

where space and time variables have been nondimensionalized using $1 / k^{*}$ and $1 / \omega^{*}$, respectively, and $a=a^{*} k^{*}$ is the dimensionless forcing amplitude. A plot of the vertical pressure gradient associated with this flow, $g(x)=-\frac{1}{4} \frac{\partial \Phi}{\partial z}(x, 0)$, is shown in the left part of figure 2. Due to the symmetry of the problem $g(x)$ is an odd function, whose characteristic extent (near both endwalls) is comparable to the container depth.

In the right part of figure 2, the regions affected by each component are indicated. As seen in this figure, the region affected by the harmonic wavetrains occupies a part of the boundary layer associated with the surface waves (its width being determined by the damping), while the region of the oscillating bulk flow occupies a part of the bulk region, with its width determined by the container depth.

Both harmonic components provide a parametric forcing mechanism through nonlinear terms in the boundary condition on the free surface (see, for instance, Miles and Henderson 1990, Higuera et al 2013). Forcing via the OBF comes from the nonhomogeneous (slowly varying in space) pressure gradient at the free surface, which is the counterpart of the spatially constant pressure gradient associated with the vertically forced Faraday problem. Forcing via harmonic wavetrains, instead, occurs through resonant triad interactions (see Hammack and Henderson 1993 and references therein) since harmonic wavetrains exhibit a lengthscale comparable to that of the (parametrically) excited subharmonic waves and spatial resonance requires a relation among the wavevectors.

In the general case, both forcing mechanisms will be present and will compete. The relative strength of each mechanism is determined by the container depth $d$ and the magnitude of the damping $C_{g}$. In the case of deep containers, the $\mathrm{OBF}$ is expected to be the dominant forcing mechanism for subharmonic instabilities unless the damping is extremely small. In this paper only forcing due to the OBF will be considered.

Both harmonic and (parametrically excited) subharmonic waves can promote a viscous mean flow, i.e., an overall circulation in the bulk region evolving on a slow timescale, induced by the shear stress associated with the propagation of gravity-capillary waves (see Higuera et al 2013 and references therein). The viscous mean flow can, in turn, modify the dynamics of surface waves. This mean flow can be incorporated in the (weakly nonlinear) analysis following the ideas in Vega et al (2004) but, since it appears at third order, it plays no role in the linear analysis considered in this paper and will therefore be ignored.

\subsection{Linear stability analysis}

For the sake of completeness a brief summary of the linear stability analysis of subharmonic waves developed by Perez-Gracia et al (2014) is presented here.

The mathematical treatment begins with the Navier-Stokes equations (with the corresponding boundary conditions, including surface tension effects) and the kinematic condition for the movement of the free surface. The first step, in accordance with the small damping assumption, is a quasipotential approximation (in the spirit of Zhang and Viñals 1997a). In this approximation certain nonlinear terms (responsible for parametric forcing) must be retained.

The flow variables are then decomposed into harmonic and subharmonic components. The subharmonic component of the free surface elevation $f_{s u b}(x, y, t)$ is written as 


$$
f_{s, t}(x, y, t)=\mathrm{e}^{\mathrm{it}} F(x, y, t)+\mathrm{c} . \mathrm{c} .,
$$

in order to factor out the fast temporal scale associated with the subharmonic oscillation (as a consequence $\left|\partial_{l} F\right| \ll|F|$ ). Recall that the wavenumber and frequency have been nondimensionalized with $k^{*}$ and $\omega^{*}$. respectively. Retaining only the linear terms in the (complex) amplitude $F$, the equation

$$
\partial_{i} F+\frac{\mathrm{i}}{4}(1+2 S)(\Delta F+F)=-2 C_{z} F+\mathrm{i} a g(x) \bar{F},
$$

is obtained, where (as stated above) $a$ is the nondimensional forcing amplitude. $g(x)$ describes the nondimensional vertical pressure gradient generated by the $\mathrm{OBF}$, and the overbar denotes complex conjugation.

A key property of the derivation of the general amplitude equation (7) is that the fast spatial scales (characterized by the subharmonic wavelength $2 \pi / k^{*}$ ) are not filtered out. As a consequence, there is no assumption of any particular pattern. The resonance condition (required in the derivation of the amplinide equation) is given by

$$
|\Delta F+F| \ll|F|,
$$

which is consistent with the fact that $|\partial F| \ll|F|$, and both $C_{g}$ and $a$ are small. In addition. appropriate boundary conditions must be imposed. These involve the reflection of surface waves at the walls of the container (reflection coefficients depend on the incident angle and thus the boundary condition involves a Fourier transform).

Due to linearity, each transverse wavelength $\kappa$ can be analyzed separately,

$$
F(x, y, t)=\left(F^{+}(x) \mathrm{e}^{\mathrm{i} \Omega t}+\bar{F}^{-}(x) \mathrm{e}^{-\mathrm{i} \Omega S t}\right) \cos (k y+\delta)
$$

where the lateral boundary condition leads to quantization (the number of allowed modes $K$ increases with $L_{2}$ ) and the separation of even and odd modes (with respect to reflection in the transverse direction). Note that using (8), both a longitudinal wavenumber $\nu$ given by $\nu=\left(1-\kappa^{2}\right)^{1 / 2}$ and an orientation of the wave pattern $\varphi$ (see figure 1) defined through $\sin (\varphi)=\nu$ can be derived from $\kappa$.

The stability of the basic state is analyzed by seeking nontrivial solutions of the system

$$
\begin{aligned}
& \mathrm{i} \Omega F^{+}+\frac{\mathrm{i}}{4}(1+2 S)\left(\partial_{x x} F^{+}+\left(1-\kappa^{2}\right) F^{+}\right)=-2 C_{g} F^{+}+\mathrm{i} a g(x) \bar{F}^{-} \\
& \mathrm{i} \Omega F^{-}-\frac{\mathrm{i}}{4}(1+2 S)\left(\partial_{x x} F^{-}+\left(1-\kappa^{2}\right) F^{-}\right)=-2 C_{g} F^{-}-\mathrm{i} a g(x) \bar{F}^{+} .
\end{aligned}
$$

with the boundary condition

$$
\partial_{x} F^{+} \pm \gamma(\nu, S) F^{+}=\partial_{x} F^{-} \pm \gamma(\nu, S) F^{-}=0 \quad \text { at } x= \pm L_{1} .
$$

This system needs to be solved numerically to determine the neutral stability curve $a(\nu)$. In Perez-Gracia et al (2014), where more details can be found, the prediction of the threshold amplinude given by this linear analysis has been compared to the experimental results by Porter et al (2012) with very good agreement. 

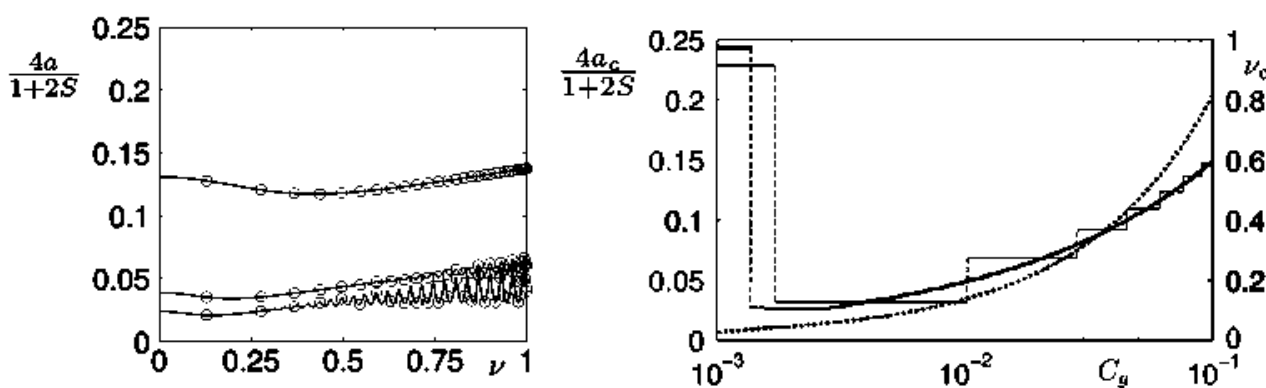

Figure 3. Left: neutral stability curves for damping values: $C_{g}=5 \times 10^{-2} \cdot C_{g}=10^{-2}$ and $C_{g}=5 \times 10^{-3}$ (from top to bottom). The values of the remaining parameters are $L_{1}=d=50$ and $S=0.5$. Modes selected by the lateral boundary condition (with $L_{2}=L_{1}$ ) are indicated by (open) circles. Right: threshold amplitude (dashed, thick line; values to be read on the left axis) and associated orientation (continuous, thick line: values on the right axes) as functions of damping, with the same parameters. Thin lines are used for threshold amplitude and associated orientation that takes mode selection by the lateral boundary condition into account.

\section{Very large (and deep) horizontally vibrated containers}

Apart from the assumptions made in section 2. we assume in this section that $L_{1} C_{3}$ is large enough that the subharmonic waves (excited by the vertical pressure gradient associated with the OBF near each endwall) are localized near the endwalls and do not interact among each other. In addition, for the sake of simplicity, it will be assumed that $L_{2}=L_{1}$ whenever $L_{2}$ is (considered) finite. In the experiments of Porter et al (2012), which used a $9 \mathrm{~cm}$ wide container. $L_{1} C_{g}$ is 1.05 with $5 \mathrm{cSt}$ silicone oil and $50 \mathrm{~Hz}$ forcing, and 2.1 with $10 \mathrm{cSt}$ oil at the same frequency. Although $L_{1} C_{\&}$ can clearly be made larger with larger containers, higher frequencies, or higher viscosities, these values are sufficient to avoid the interaction of opposing wavetrains for most practical purposes (since the exponential decay of the subharmonic waves determined by equation (10) is relatively fast).

\subsection{Effect of parameters on threshold amplitude and orientation}

To illustrate the response of the system. a representative case with $L_{1}=L_{2}=d=50$ is taken and the effect of the different parameters is investigated. Note that, in spite of the OBF extending into the interior when $d=L_{1}$, the interaction between subharmonic surface waves generated at each endwall (at threshold) can still be weak because the nonuniform forcing $g(x)$ is much smaller in the center of the container than at the endwalls (see figure 2).

Figure 3 illustrates the effect of damping. As seen below, this is nearly the only sensitive parameter in this limit. The neutral stability curve $a(\nu)$ (including the effect of quantization by the lateral boundary condition) is shown at the left part for the indicated values of $C_{g}$. The resulting threshold amplitude. defined by locating the minimum of the neutral stability curve. and its associated orientation (characterized by the longitudinal wavelength $\nu$ ) are shown in the right part of this figure. Both the threshold amplitude and orientation vary monotonically with the damping $C_{g}$. except at very low values (in this case $L_{1} C_{z}$ is no longer sufficiently large and some interaction between subharmonic surface waves generated at opposing endwalls will occur, as considered in the next section). A smooth transition is observed from nearly 

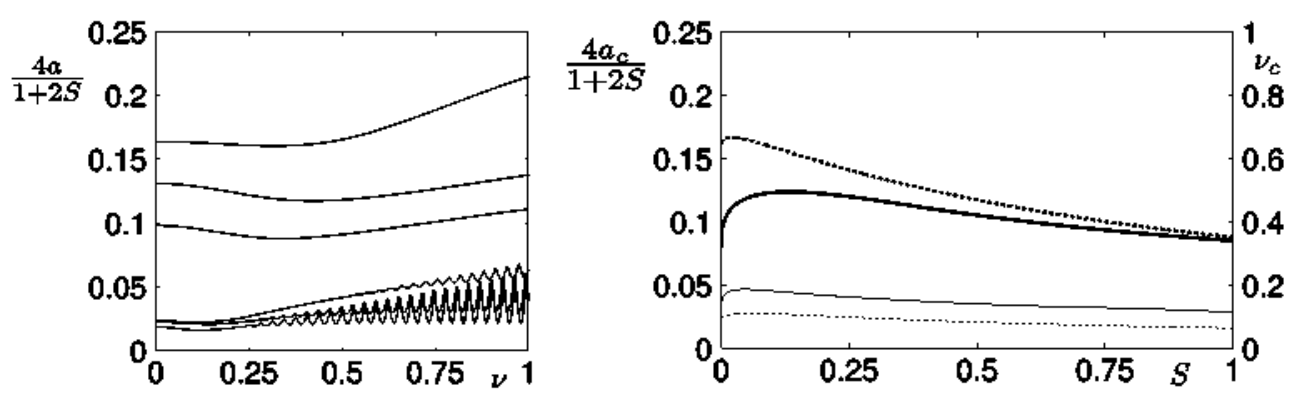

Figure 4. Left: neutral stability curves for $C_{g}=5 \times 10^{-3}$ (lower three curves) and $C_{\mathrm{g}}=5 \times 10^{-2}$ (upper three curves) for three values of the gravity-capillary balance $S$ : $0,0.5$ and $1(S=0$ corresponds to the highest curve in each group and $S=1$ to the lowest one). All calculations are with $L_{1}=d=50$. Right: variation of threshold amplitude (discontinuous lines, left axis) and orientation (continuous lines, right axis) with the gravity-capillary balance $S$ for $L_{1}=d=50$ and damping $C_{g}=5 \times 10^{-3}$ (thin lines) and $C_{g}=5 \times 10^{-2}$ (thick lines).
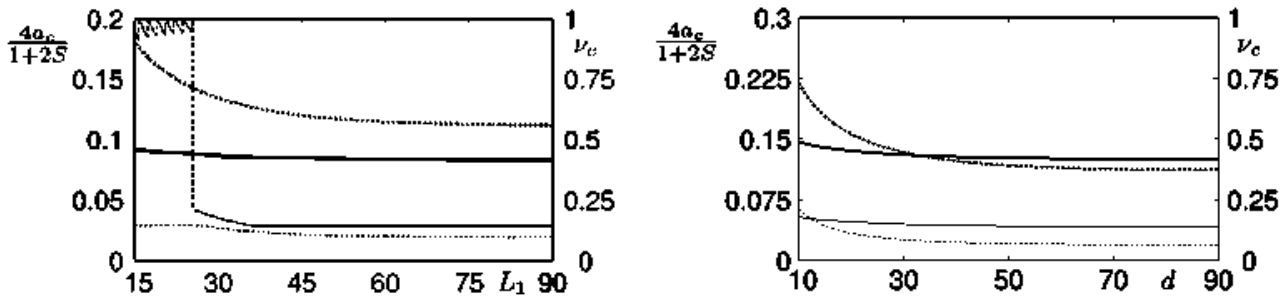

Figure 5. Left: variation of threshold amplitude (discontinuous lines, left axis) and orientation (continuous lines, right axis) with container length $L_{1}$ for $S=0.5, d=50$ and damping $C_{g}=5 \times 10^{-3}$ (thin lines) and $C_{g}=5 \times 10^{-2}$ (thick lines). Right: variation of threshold amplitude and orientation with container depth $d$ for $L_{1}=50, S=0.5$ and $C_{\mathrm{g}}=5 \times 10^{-3}$ and $C_{\mathrm{g}}=5 \times 10^{-2}$ (curve styles signify the same thing as in the left plot).

crosswise orientation at low (but not too low) damping to more rotated (but still far from parallel) waves at larger values of damping. The threshold amplinde, as expected, increases with damping.

When $L_{2}$ is assumed to be finite, a large number of allowed modes (marked by open circles) can be seen in figure 3 because $L_{2}=L_{1}$ is large. As a result of this, and the fact that the minimum of the neutral stability curve is located in the regular (nonoscillating) part of the curve, which corresponds to low values of the longitudinal wavenumber $\nu$ in the case of small damping. the effect of quantization on the selection of both the threshold value of the amplinude $a$ and the orientation $\nu$ is weak: for the sake of clarity, it will not be shown in the remaining figures in this subsection.

The effect of the gravity-capillary balance $S$ is shown in figure 4. Again, both the critical amplinude and orientation vary monotonically (and slowly) with $S$ except at very small values near the pure gravity wave limit. In this limit (which is singular, since reflection conditions should be reformulated in the absence of surface tension) a small deviation from pure crosswaves is also observed. 
The effect of $L_{1}$ and of $d$ are both weak. Figure 5 shows that when $L_{1}$ is increased beyond about 40 the limiting case of an infinitely large container applies. If $L_{1}$ is not large then more complex dependence is observed. However, in this case $L_{1} C_{8}$ is no longer large and interaction between subharmonic waves generated at opposing endwalls is expected (considered in the next section). Finally, the effect of $d$ is only apparent for the threshold amplitude and this dependence is very weak except with relatively small values of $d$. The orientation of the pattern is nearly independent of $d$.

The threshold frequency $\Omega_{c}$, which measures the deviation from $2: 1$ resonance, is shown in figure 6. Although it is generically nonzero-the threshold value $\Omega_{c}$ is exactly zero only for particular combinations of the parameters-its value is very small except when $L_{1}$ is small (which. again, is the case considered in the next section). Thus, although the instability is in general associated with a finite (Hopf) frequency and quasiperiodic motion, in this limit it will appear, to a high degree of accuracy, as a pure $2: 1$ resonance.

\subsection{Onset modes and dynamics}

Equation (7) is equivariant under the spatiotemporal symmetry: $x \rightarrow-x, F \rightarrow \mathrm{i} F$, which describes a reflection in $x$ (recall that the forcing $g(x)$ is odd) and a simultaneous phase shift of $\pi / 2$ in time. The limit of infinitely large containers, $L_{1} C_{8} \rightarrow \infty$, possesses an additional symmetry because the waves on either side of the container are then independent and may be separately reflected (shifted by a phase $\pi$ ). The two equivalent parametrically forced systems at either endwall differ only by a temporal phase and undergo a steady state (2:1 resonant) instability at the same critical forcing value. The full system thus possesses a pair of zero eigenvalues and a two-dimensional center eigenspace, which includes both one-sided and two-sided eigenfunctions (see figures 7 and 8).

When $L_{1} C_{8}$ is finite but large, so that interaction between waves near both endwalls is weak, then arguments based on weak symmetry-breaking apply. The double zero eigenvalue of the uncoupled problem becomes a pair of imaginary eigenvalues, associated with a slowly modulated solution that cycles between (nearly) one-sided and two-sided states. On the fast timescale, the phase difference between oscillations at either end will be approximately $\pm \pi / 2$ : this phase difference changes sign during the part of the slow oscillation when the amplitude at one of the two sides is passing through zero.

In addition to the appearance of slow temporal modulation when $L_{1}$ is large but finite, one expects complex dynamics related to transverse mode interaction when $L_{2}$ is large. There will then be many possible transverse modes near the minimum of the neutral stability curve, with nearly the same threshold. Weakly nonlinear interaction between these modes introduces transverse modulation as shown in figure 9. The complicating effects of mode interaction will be enhanced further when $C_{g}$ is small because there will then be multiple instability tongues with almost the same threshold (the term 'tongue' refers here to the sharp but continuous oscillations in the neutral stability curve as shown in figures 3 and 4).

\section{Moderately large (and deep) horizontally vibrated containers}

In addition to the assumptions made in section 2 , we consider in this section a large container where the damping $C_{g}$ is small enough that $L_{1} C_{g}$ is moderate or even small. This results in a moderate or large interaction between the waves excited at each endwall. This limit could, for example, be reached experimentally with a $9 \mathrm{~cm}$ square container of $5 \mathrm{cSt}$ oil by using very 


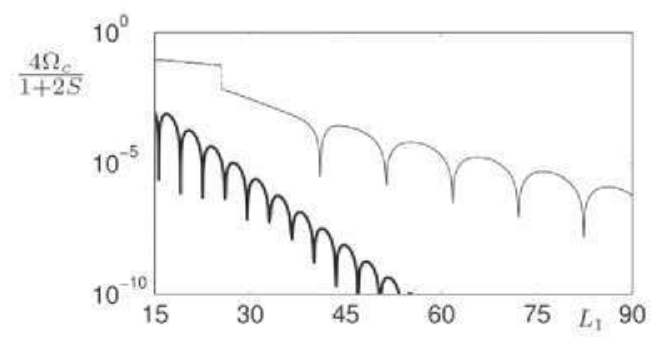

Figure 6. Variation of frequency at threshold $\Omega_{c}$ with $L_{1}$ for $d=50$ and $S=0.5$. Two curves are plotted corresponding to $C_{g}=5 \times 10^{-3}$ (thin line) and $C_{g}=5 \times 10^{-2}$ (thick line).
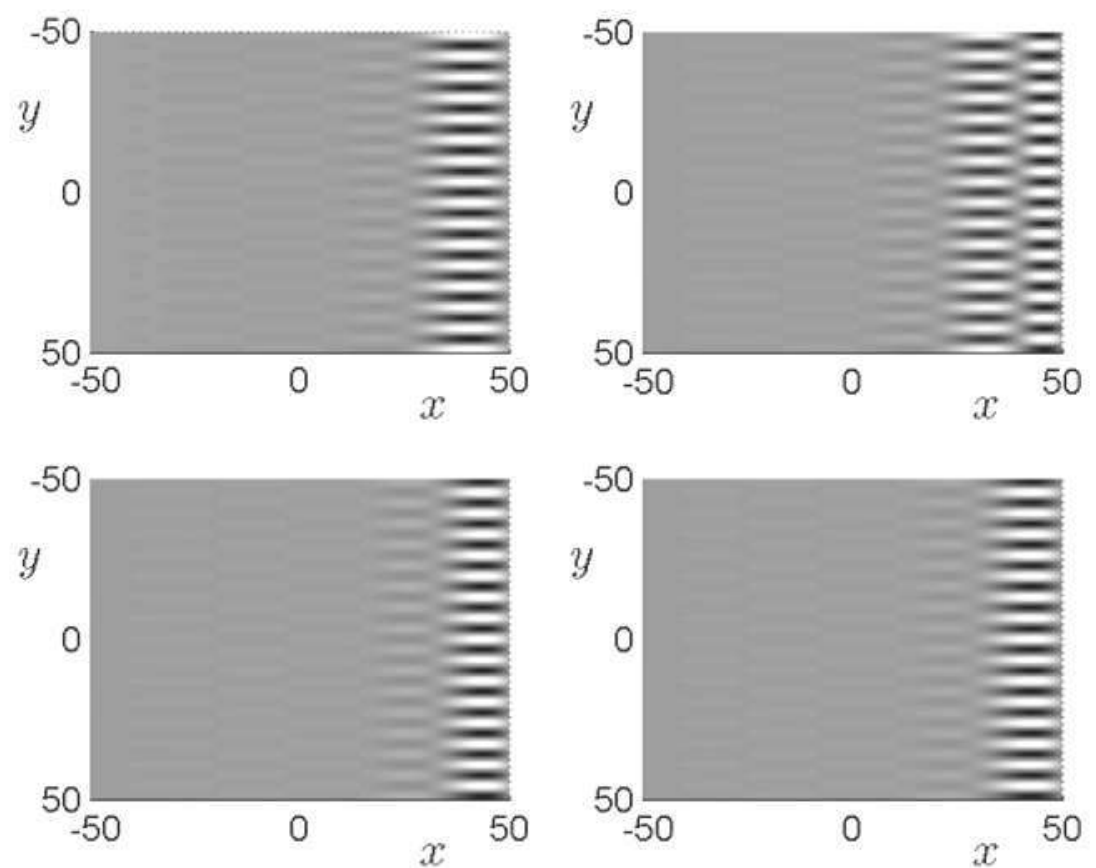

Figure 7. Elevation of the free surface associated with the subharmonic waves, $f_{s u b}(x, y, t)$, described by a one-sided eigenfunction at $t=0$ (top left), $t=\pi / 4$ (top right), $t=\pi / 2$ (bottom left) and $t=3 \pi / 4$ (bottom right) for the case considered in figure 3 with $C_{g}=0.01$.

low frequencies (less than $20 \mathrm{~Hz}$, say, where $L_{1} C_{g} \simeq 0.124$ ). Alternatively, this limit could be reached in the same container by using (pure) water (where $L_{1} C_{g}<0.18$ for frequencies less than $100 \mathrm{~Hz}$ ). As an illustrative case for this section, we take a container with $L_{1}=L_{2}=25$ and $d=10$. Damping will be varied to achieve different levels of interaction between the waves generated at both endwalls. 

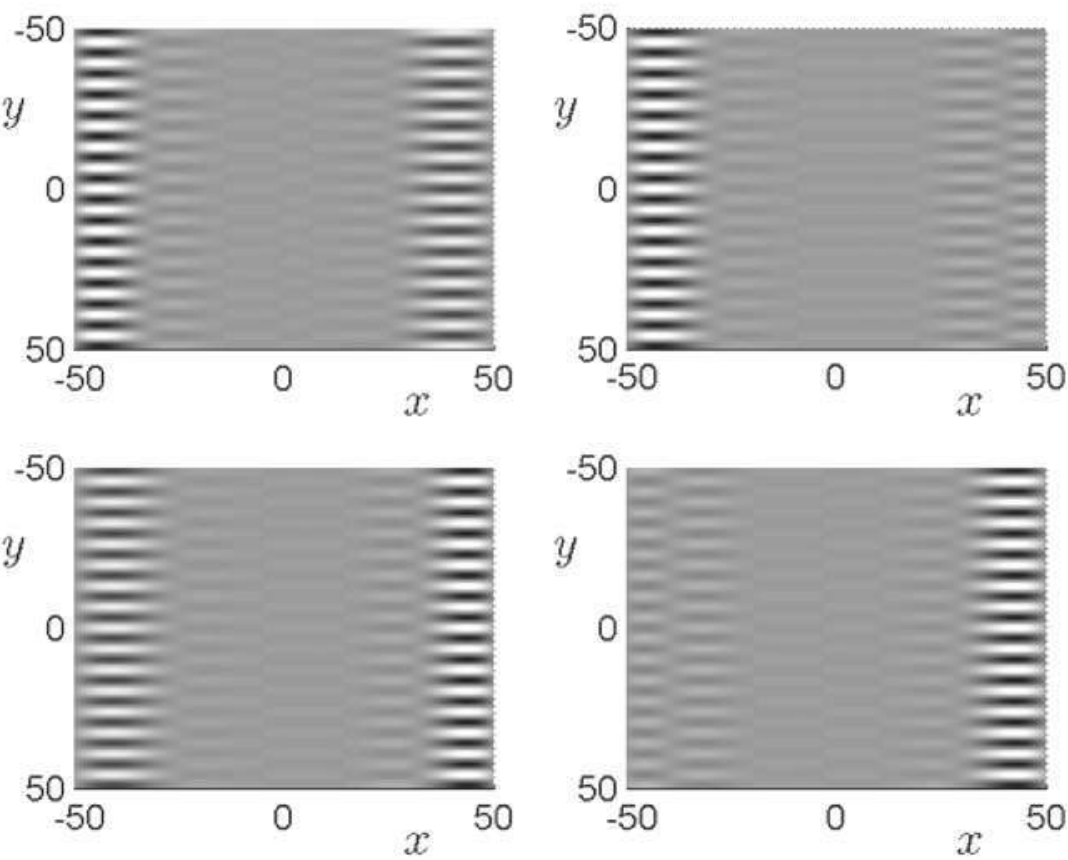

Figure 8. Counterpart of figure 7 for a two-sided eigenfunction.
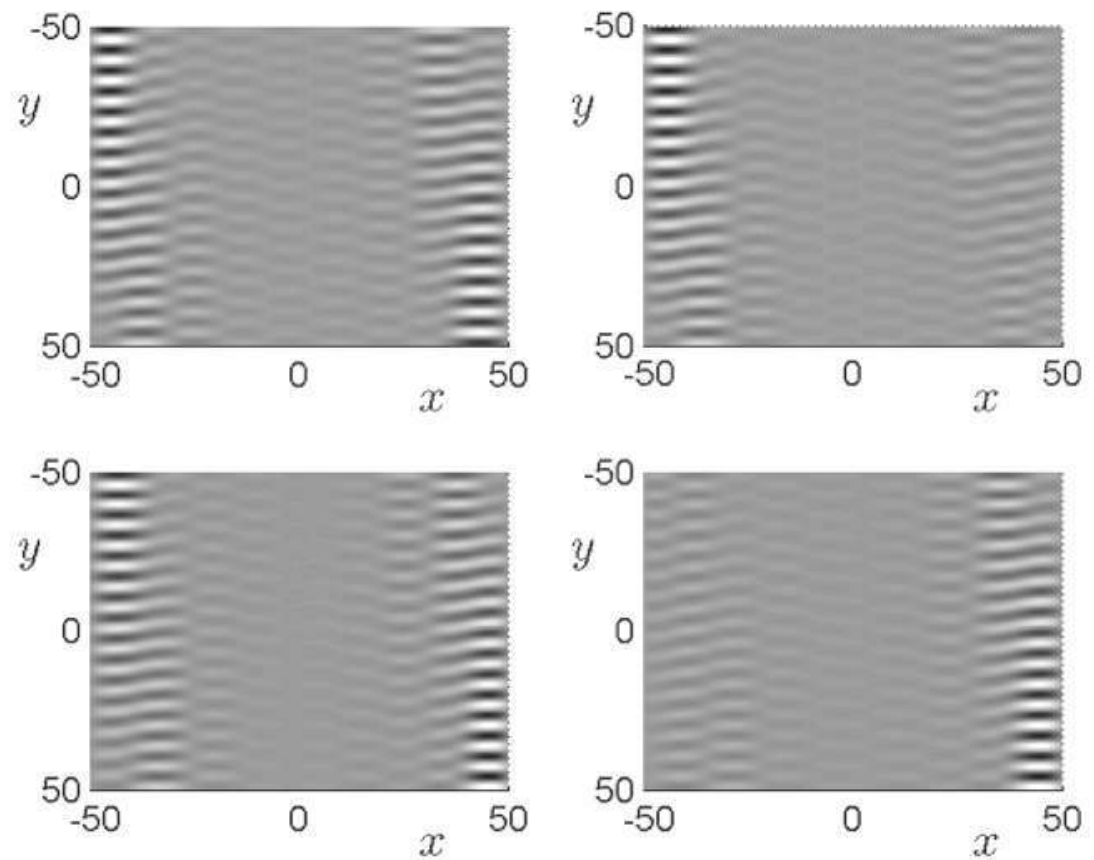

Figure 9. Counterpart of figure 7 showing the pattern arising from weakly nonlinear interaction between the first two modes (smallest threshold amplitude) on the neutral stability curve in figure 3 with $C_{g}=0.01$. 

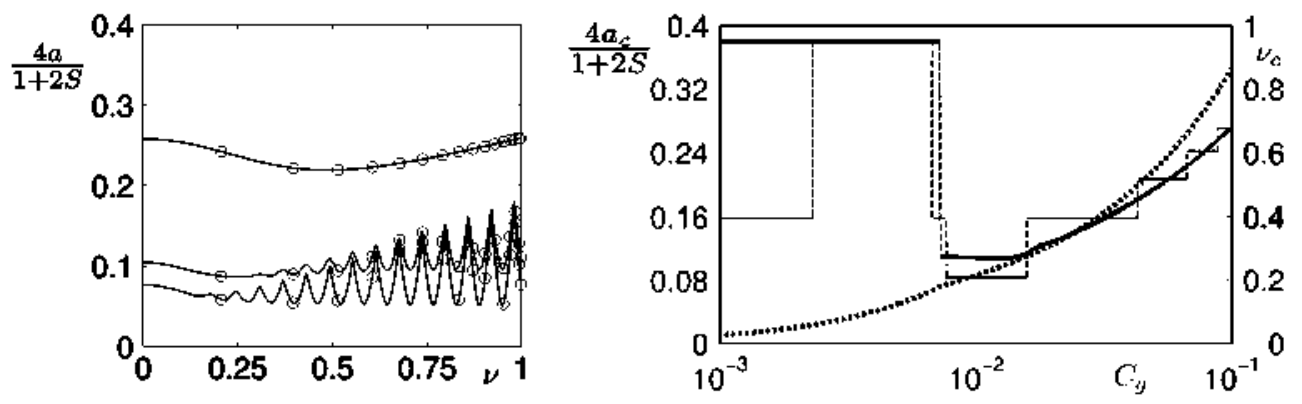

Figure 10. Counterpart of figure 3 with $L_{1}=L_{2}=25$ and $d=10$.
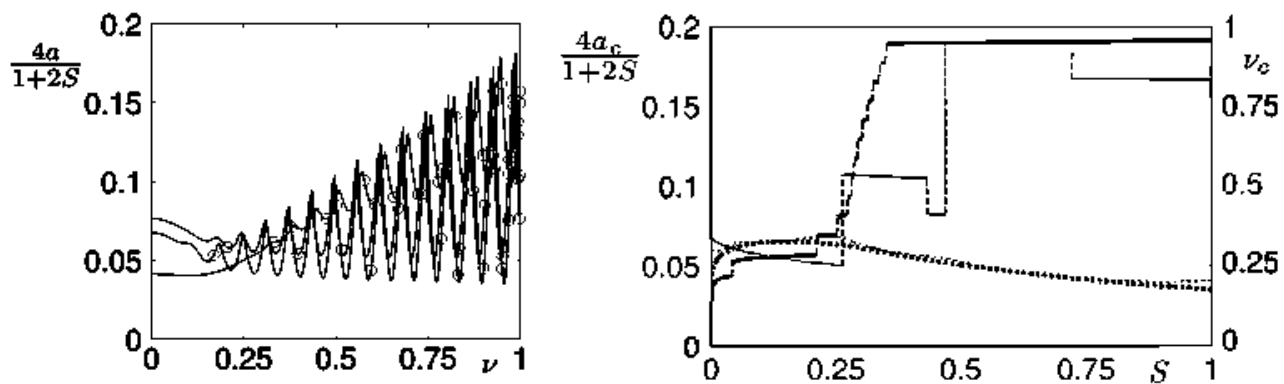

Figure 11. Left: neutral stability curves with $C_{8}=5 \times 10^{-3}, L_{1}=L_{2}=25$, and $d=10$ for three values of the gravity-capillary balance $S: 0,0.5$ and 1 . Modes selected by the lateral boundary condition (with $L_{2}=L_{1}$ ) are indicated by (open) circles. Right: variation of threshold amplitude (discontinuous thick line, left axis) and orientation (continuous thick line, right axis) with the gravity-capillary balance $S$ for $L_{1}=25$. $d=10$ and $C_{z}=5 \times 10^{-3}$. Thin lines are used for threshold amplitude and associated orientation that takes mode selection by the lateral boundary condition into account.

\subsection{Effect of parameters on threshold amplitude and orientation}

The effect of damping $C_{g}$ is shown in figure 10. Although the trend is broadly similar to figure 3 of the previous section, some important differences arise. The appearance of nearly parallel waves encountered in the previous section for very small $C_{8}$ values (due to the interaction between waves near the two endwalls) occurs in this case for a wider range of damping values. At still larger values of damping. the dependence is similar to before. Additionally, there is a strong effect of quantization in this case, which noticeably influences the orientation. In the case of very small damping this quantization effect is dramatic. With $C_{g}=10^{-3}$, for instance, the orientation changes from $\nu \simeq 0.95$ in the infinite $L_{2}$ case to $\nu \simeq 0.4$ for $L_{2}=L_{1}=25$. This is related to the appearance (for very small $C_{8}$ ) of a large number of tongues with very similar amplitude (which. in addition. can be expected to produce mode interaction near threshold).

The effect of the gravity-capillary balance $S$ is shown in figure 11 for $C_{g}=5 \times 10^{-3}$ and, in contrast with the case of the previous section (see figure 4), this effect is now strong. The dependence observed for the threshold amplitude is not dissimilar to that seen in the previous section (although quantization effects lead to an irregular curve), but the dependence of 

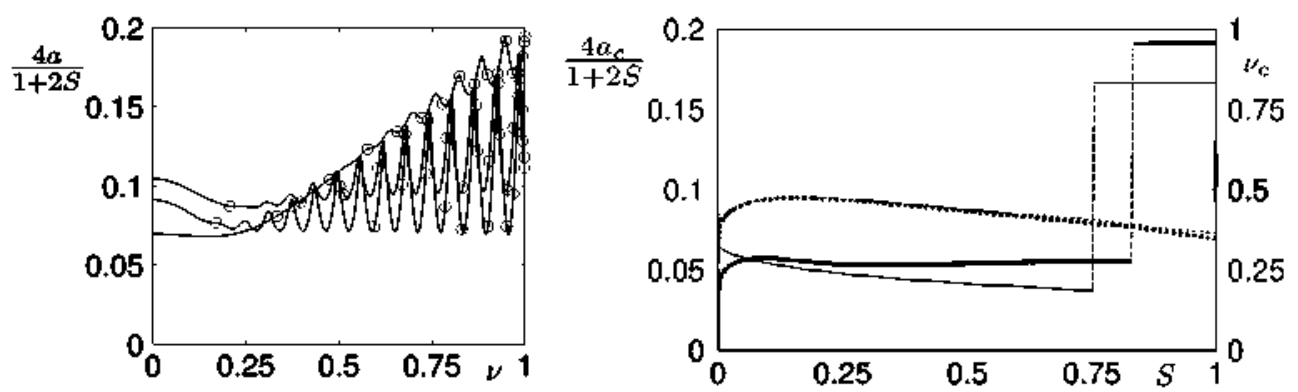

Figure 12. Counterpart of figure 11 for $C_{\mathrm{g}}=10^{-2}$.
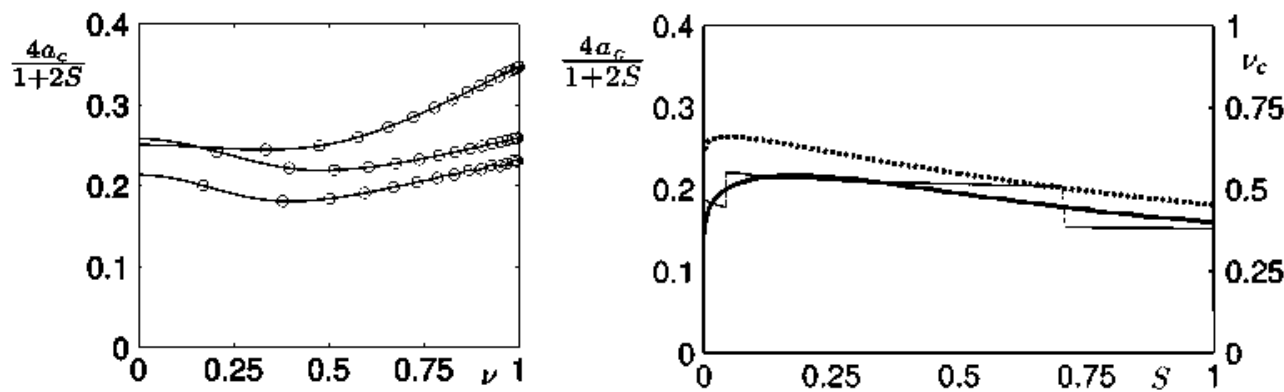

Figure 13. Counterpart of figure 11 for $C_{g}=5 \times 10^{-2}$.
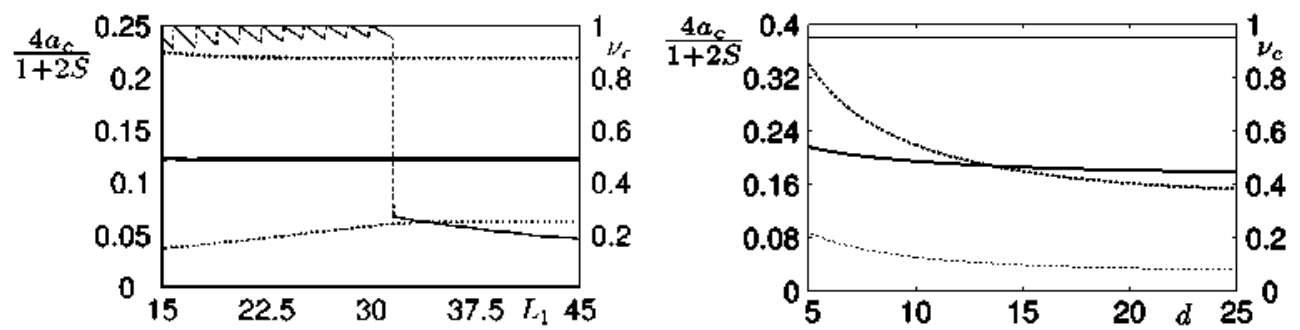

Figure 14. Counterpart of figure 5 for $C_{g}=5 \times 10^{-3}$ and $C_{g}=5 \times 10^{-2}$ with $L_{1}=25$ and $d=10$.

threshold orientation on $S$ is much different. In the case of infinite $L_{2}$, waves rotate from (almost pure) cross-waves in the gravity wave limit to nearly parallel waves in the capillary wave limit, passing through oblique angles (with jumps associated with transitions from one tongue to another). Quantization effects when $L_{2}=L_{1}$ produce additional new features, including different behaviour in the gravity wave limit where oblique waves are observed instead of cross-waves. As shown in figures 12 and 13, this effect becomes weaker for larger values of damping (as interaction between waves at both endwalls decreases).

The effect of the depth $d$, which is shown in figure 14 , is similar to the case of large containers (figure 5). The effect of $L_{1}$, in contrast. can be quite different with small damping (when strong interaction between waves originating at opposing endwalls is expected). If $L_{1}$ is not large enough, the threshold amplitude will correspond to the closest tongue to $\nu=1$ (see figures 10 and 11). Jumps from one tongue to another are expected as $L_{1}$ is varied (these are 

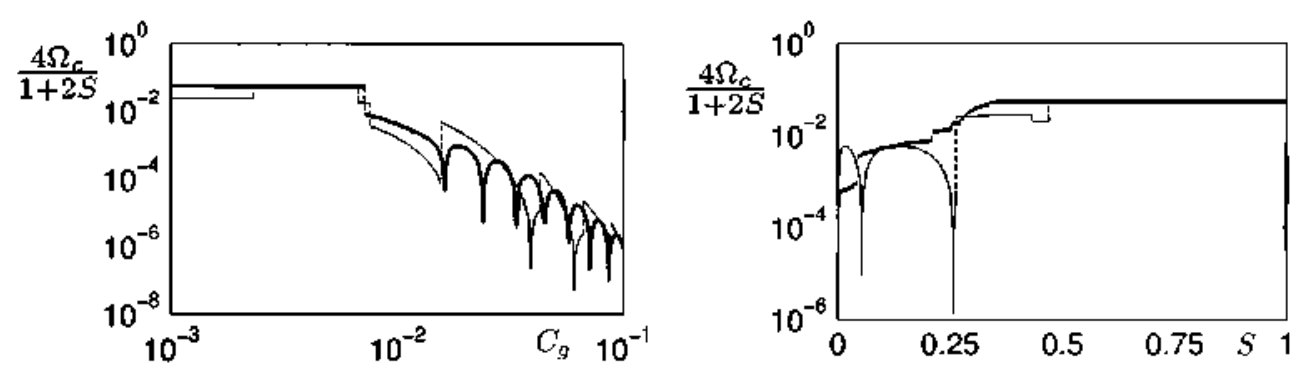

Figure 15. Left: frequency at threshold $\Omega_{c}$ as a function of damping $C_{g}$ with $L_{1}=L_{2}=25, d=10$ and $S=0.5$. Right: frequency at threshold $\Omega_{c}$ as a function of $S$ for $C_{g}=5 \times 10^{-3}$. Thick (thin) lines correspond to $L_{1}=25$ and infinite (finite) $L_{2}$.
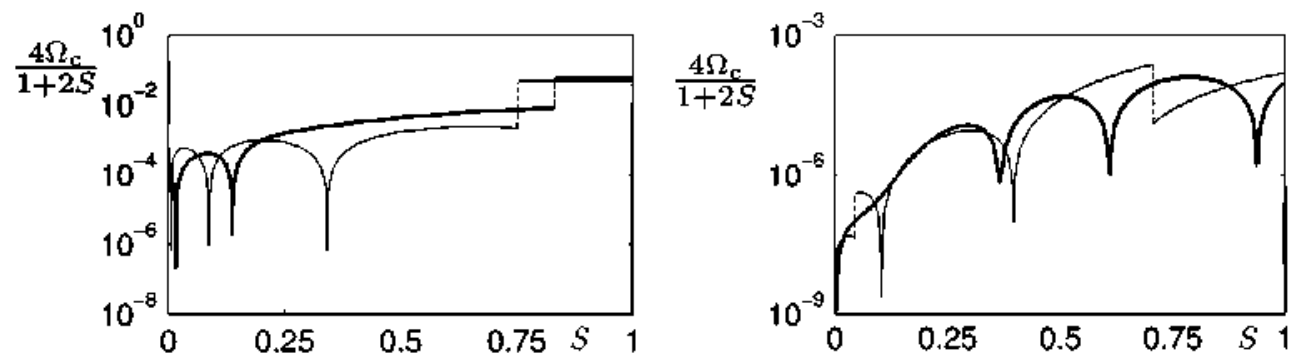

Figure 16. Counterpart of the right part of figure 15 for $C_{\mathrm{g}}=10^{-2}$ (left) and $C_{\mathrm{y}}=5 \times 10^{-2}$ (right).

clearly seen in figure 14). Quantization effects (not shown in figure 14 for the sake of clarity) lead to a more complex dependence of the orientation on $L_{1}$. It is worth noting again that. in such cases where the successive tongues have similar minimum amplitudes, one expects interaction between a number of modes near onset, both in the finite and infinite $L_{2}$ case.

\subsection{Subharmonic or quasiperiodic instability?}

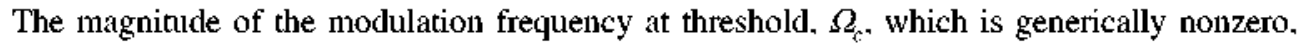
depends strongly on damping. The left part of figure 15 shows the frequency $\Omega_{c}$ as a function of damping. It is clear that, when there is strong interaction between the waves coming from opposite endwalls, its magnitude is not so small. and this fact is irrespective of quantization effects, as seen in the figure.

The right part of figure 15 shows the frequency $\Omega_{\text {a }}$ as a function of the gravity-capillary balance $S$ for $C_{8}=5 \times 10^{-3}$. In the gravity wave limit (keeping in mind that this limit is singular in the present analysis), the frequency $\Omega_{c}$ is close to zero (this is consistent with the focus of previous theoretical analyses on pure subharmonic cross-waves). As $S$ increases and the pattern orientation varies from nearly crosswise to nearly parallel (see figure 11 ), the

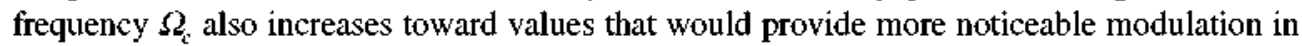
a practical. experimental context. As seen in the same figure, when $L_{2}$ is finite the results are not qualitatively different. except for the appearance of (pure) 2:1 resonance at two particular values of $S$; when $L_{2}$ is infinite, these pure 2:1 resonances do not appear since threshold 
values occur at the minima of one of the tongues and the frequency $\Omega$ is always maximal at these points (see Perez-Gracia et al 2014). In practice, at such small values of the damping, complicated behaviour will be expected near onset since (in addition to the competition with triad resonances) the interacting modes associated with different tongues possess not only different longitudinal wavelengths but different frequencies as well. Quantization effects due to finite $L_{2}$ would add still more complexity to the potential interactions.

For larger values of the damping $C_{g}$, a comparatively simpler scenario occurs. Figure 16 shows the results for $C_{g}=10^{-2}$ and $C_{g}=5 \times 10^{-2}$. In these cases, except near the capillary limit for $C_{g}=10^{-2}$, the threshold is associated with a small longitudinal wavelength (not with oblique waves) and the frequency varies smoothly and can be zero for particular values of $S$. The gravity wave limit, in particular, seems to be associated with a zero frequency when $L_{2}$ is infinite. Quantization effects due to finite $L_{2}$ produce (slight) quantitative changes, like a nonzero frequency in the gravity limit (but the associated frequency is still very small).

\section{Concluding remarks}

The parametric excitation of subharmonic surface waves on the free surface of a horizontally vibrated container has been considered under the assumptions of a large and deep container (compared to the wavelength of the waves). Weak damping was also assumed so that surface wave propagation could be considered to be nearly inviscid at the characteristic lengthscale given by the wavelength; damping along the entire length of the container can, nonetheless, be significant. Two parametric forcing mechanisms were identified, one associated with the directly forced harmonic wavetrains produced at the endwalls and one associated with the $\mathrm{OBF}$. The container was assumed to be deep enough that the second mechanism dominates. The linear analysis developed by the authors (Perez-Gracia et al 2014) was then used to systematically investigate the role of the different parameters.

Since the product of the container length $L_{1}$ and the damping parameter (Ohnesorge number) $C_{g}$ controls the strength of the interaction between subharmonic waves generated at opposing endwalls, two limits of weak and significant interaction have been analyzed considering (moderately) large and small values of $L_{1} C_{g}$, respectively. A central result of this analysis is the importance of the interaction in producing quasiperiodic solutions at onset. This is a very general effect of interacting waves produced by out-of-phase forcing mechanisms that can be understood as a result of symmetry-breaking in the weakly interacting limit.

In the limit of large containers and/or moderate (not too small) damping, the product $L_{1} C_{8}$ is large enough that interaction between the subharmonic surface waves at each endwall is negligible. This is the relevant limit, for example, in experiments (Porter et al 2012) with $2 L_{1}^{*}=2 L_{2}^{*}=9 \mathrm{~cm}$ and $d^{*}=5 \mathrm{~cm}$ using silicone oil (with kinematic viscosities of $5 \mathrm{cSt}$ or greater) and forcing frequencies above $50 \mathrm{~Hz}$ since, in these cases, $L_{1} C_{g}>1$. The instability in this limit is always close to pure $2: 1$ resonance (i.e., the modulation frequency is extremely small). In this regime we find that parameters other than damping have little influence on the threshold amplitude and pattern orientation. In the gravity wave limit, the orientation of the surface waves at threshold is nearly crosswise, as observed in many wavemaker experiments (see Miles and Henderson 1990 and references therein).

In the limit of moderately sized containers and/or small values of damping, the product $L_{1} C_{g}$ is not large and interaction between the subharmonic surface waves at each endwall 
cannot be neglected. There is a noticeable deviation from pure 2:1 resonance in this case, particularly when capillary effects dominate, leading to quasiperiodic solutions at onset. This limit can be reached experimentally using lower frequencies or lower viscosities. With the same $9 \mathrm{~cm}$ square container, for example. driving frequencies of $20 \mathrm{~Hz}$ or less will give $L_{1} C_{g}<0.13$ with $5 \mathrm{cSt}$ silicone oil. while driving frequencies of less than $100 \mathrm{~Hz}$ will give $L_{1} C_{g}<0.18$ with (clean) water. In addition to damping, the gravity-capillary balance and the presence of lateral walls have an important effect on threshold amplitude and (especially) on pattern orientation in this regime. In the gravity wave limit the orientation is again nearly crosswise, while in the capillary wave limit the waves prefer an orientation that is nearly parallel to the vibrating endwalls. In any case. the selected orientation is strongly affected by the presence of lateral walls (quantization).

It is important to bear in mind that the predictions described here are the results of a linear analysis. While (predicted) threshold amplitudes are observable in experiments, the predicted properties of the onset patterns (like spatial orientation and temporal modulation) may or may not be seen in a given experiment. This depends to a large extent on whether the bifurcation to subharmonic waves is supercritical or not. Even in the case of supercritical bifurcation. complicated dynamics can be anticipated near onset due to mode interaction. In the case of very wide containers $\left(L_{2} \gg 1\right)$ there will be many modes with similar threshold amplinides and much care must be taken to detect simple (pure mode) patterns; otherwise, interaction between modes with similar orientation (and modulation frequency $\Omega$ ) will lead to (laterally) modulated patterns. Even in the case when $L_{2}$ is not so large, several modes can (for particular combinations of the parameters) exhibit similar threshold amplitudes and the interaction between these distinct modes would again lead to very complicated patterns. The temporal modulations that arise when $L_{1} C_{g}$ is finite (i.e.. not too large) are expected to be observable in some cases (experimental confirmation of this is currently being undertaken by one of us (JP) and will be described elsewhere). In addition to the requirement of supercriticality. the interaction between opposing wavetrains must be large enough that $\Omega$ is not excessively small. With a driving frequency of $50 \mathrm{~Hz}$, for example, a Hopf frequency $\Omega$ of $10^{-3}$ would give a modulation period of about $40 \mathrm{~s}$. Modulation periods of significantly more than this would be difficult to measure accurately. Taking this value for a reference. figures 6 and 15 suggest $\Omega>10^{-\hat{3}}$ requires $L_{1} C_{\mathrm{g}} \lesssim 0.2$.

Despite anticipated complications due to nonlinear effects, we expect the results in this paper to provide another step in the understanding of free surface dynamics in horizontally vibrated containers, as well as a useful guide for experimentalists in this field.

\section{Acknowledgments}

This research was partially supported by the Spanish Ministry of Science and Innovation, under Grants TRA2010-18054 (JMPG and JMV), MTM-2010-21135-C02-02 (FV) and AYA2010-19081 (JP).

\section{References}

Ayanle H, Bernoff A J and Lichter S 1990 Spanwise modal competition in cross-waves Physica D 43 87-104

Barnard B J S and Pritchard W G 1972 Cross-waves Part 2 Experiments J. Fluid Mech. 55 245-55 
Bernoff A J, Kwok L P and Lichter S 1989 Viscous cross-waves: an analytical treatment Phys. Fluids A $1678-88$

Bestehorn M, Han Q and Oron A 2013 Nonlinear pattern formation in thin liquid films under external vibrations Phys. Rev. E 88023025

Faraday M 1831 On a peculiar class of acoustical figures; and on certain forms assumed by groups of particles upon vibrating elastic surfaces Phil. Trans. R. Soc. Lond. 121 299-340

Feng Z C 1997 Transition to traveling waves from standing waves in a rectangular container subjected to horizontal excitations Phys. Rev. Lett. 79 415-8

Funakoshi M and Inoue S 1988 Surface waves due to resonant horizontal oscillation J. Fluid Mech. 192 $219-47$

Garrett C J R 1970 On cross-waves J. Fluid Mech. 41 837-49

Gresho P M and Sani R L 1970 The effects of gravity modulation on the stability of a heated fluid layer J. Fluid Mech. $40783-806$

Hammack J L and Henderson D M 1993 Resonant interactions among surface water waves Annu. Rev. Fluid Mech. 25 55-97

Higuera M, Porter J, Varas F and Vega J M 2013 Nonlinear dynamics of confined liquid systems with interfaces subject to forced vibrations Adv. Colloid Interface Sci. 206 106-15

Ivanova A A, Kozlov V G and Tashkinov S I 2001 Interface dynamics of immiscible fluids under circularly polarized vibration (experiment) Fluid Dyn. $360871-9$

Jones A F 1984 The generation of cross-waves in a long deep channel by parametric resonance $J$. Fluid Mech. 138 53-74

Kit E and Shemer L 1989 On the neutral stability of cross-waves Phys. Fluids A 1 1128-32

Lichter S and Bernoff A J 1988 Stability of steady cross waves: theory and experiment Phys. Rev. A 37 $1663-7$

Lichter S and Chen J 1987 Subharmonic resonance of nonlinear cross-waves J. Fluid Mech. 183 451-65

Lin J D and Howard L N 1960 Non-linear standing waves in a rectangular tank due to forced oscillation Hydrodynamics Laboratory Technical Report 44, MIT

Miles J and Henderson D 1990 Parametrically forced surface waves Annu. Rev. Fluid Mech. 22 $143-65$

Miles J W 1984 Resonantly forced surface waves in a circular container J. Fluid Mech. 149 15-31

Moisy F, Michon G-J, Rabaud M and Sultan E 2012 Cross-waves induced by the vertical oscillation of a fully immersed vertical plate Phys. Fluids 24022110

Nicolas J A, Rivas D and Vega J M 1998 On the steady streaming flow due to high-frequency vibration in nearly inviscid liquid bridges J. Fluid Mech. 354 147-56

Perez-Gracia J M, Porter J, Varas F and Vega J M 2014 Subharmonic capillary-gravity waves in large containers subject to horizontal vibrations J. Fluid Mech. 739 196-228

Porter J, Tinao I, Laverón-Simavilla A and Lopez C A 2012 Pattern selection in a horizontally vibrated container Fluid Dyn. Res. 44065501

Schuler M 1933 Der umschlag von oberflächenwellen Zeitschrifg für Angew. Math. u. Mech. 13 443-6

Shemer L and Kit E 1989 Long-time evolution and regions of existence of parametrically excited nonlinear cross-waves in a tank J. Fluid Mech. $209249-63$

Snoeijer J H and Andreotti B 2013 Moving contact lines: scales, regimes, and dynamical transitions Annu. Rev. Fluid Mech. 45 269-92

Talib E, Jalikop S V and Juel A 2007 The influence of viscosity on the frozen wave instability: theory and experiment $J$. Fluid Mech. $58445-68$

Taneda S 1994 Visual observations of the flow around a half-submerged oscillating circular cylinder Fluid Dyn. Res. 13 191-151

Topaz C M, Porter J and Silber M 2004 Multifrequency control of Faraday wave patterns Phys. Rev. E 73066206

Underhill W B, Lichter S and Bernoff A J 1991 Modulated, frequency-locked, and chaotic cross-waves J. Fluid Mech. 225 371-94

Varas F and Vega J M 2007 Modulated surface waves in large-aspect-ratio horizonally vibrated containers J. Fluid Mech. 579 271-304

Vega J M, Knobloch E and Martel C 2001 Nearly inviscid Faraday waves in annular containers of moderately large aspect ratio Physica D 154 313-36 
Vega J M, Rüdiger S and Viñals J 2004 Phenomenological model of weakly damped Faraday waves and the associated mean flow Phys Rev E Stat Nonlin Soft Matter Phys. 70046306

Wolf G H 1969 The dynamic stabilization of the Rayleigh-Taylor instability and the corresponding dynamic equilibrium Z. Physik 227 291-300

Zhang W and Viñals J 1997a Pattern formation in weakly damped parametric surface waves $J$. Fluid Mech. 336 301-30

Zhang W and Viñals J 1997b Pattern formation in weakly damped parametric surface waves driven by two frequency components J. Fluid Mech. $341225-44$ 